\title{
BMJ Open Primary prevention of diabetic retinopathy with fibrates: a retrospective, matched cohort study
}

\author{
Christopher LI Morgan, ${ }^{1}$ David R Owens, ${ }^{2}$ Patrick Aubonnet, ${ }^{3}$ Emma S M Carr, ${ }^{3}$ \\ Sara Jenkins-Jones, ${ }^{4}$ Chris D Poole, ${ }^{1}$ Craig J Currie ${ }^{1}$
}

To cite: Morgan $\mathrm{CL}$, Owens DR, Aubonnet $\mathrm{P}$, et al. Primary prevention of diabetic retinopathy with fibrates: a retrospective, matched cohort study. BMJ Open 2013;3:e004025 doi:10.1136/bmjopen-2013004025

- Prepublication history for this paper is available online. To view these files please visit the journal online (http://dx.doi.org/10.1136/ bmjopen-2013-004025).

Received 13 September 2013 Revised 23 October 2013 Accepted 24 October 2013

CrossMark

For numbered affiliations see end of article.

Correspondence to Dr Craig J Currie; currie@cardiff.ac.uk

\section{ABSTRACT}

Objectives: To compare the progression of diabetic retinopathy (DR) in people with type 2 diabetes treated with fibrates with that of non-exposed controls.

Design: Retrospective, matched cohort study.

Setting: UK Clinical Practice Research Datalink (CPRD).

Participants: 5038 people with type 2 diabetes with a history of fibrate exposure but without evidence of DR were identified. Three thousand one hundred and seventy-six $(63 \%)$ people could be randomly matched to one non-exposed control; of these, 2599 (81.8\%) were matched without any missing blood pressure or glycated haemoglobin (HbA1c) values.

Main outcome measures: The primary endpoint was first recorded DR with a secondary endpoint of allcause mortality or first DR. Time to clinical endpoints was compared using Cox proportional hazards models. Results: Mean follow-up was 5.1 and 5.0 years for fibrate-exposed and non-exposed patients, respectively. For fibrate-exposed participants, there was a reduction in DR: 33.4 events/1000 person-years vs 40.4 $(p=0.002)$, and in death or DR: 50.6 vs 60.2 $(p<0.001)$. For those matched with full systolic blood pressure and $\mathrm{HbA} 1 \mathrm{C}$ data, crude event rates were 34.3 versus 43.9 for $D R(p<0.001)$ and 51.2 vs 63.4 $(p<0.001)$ for death or DR. Following adjustment, DR was significantly delayed for those treated with fibrates, with an adjusted HR (aHR) of $0.785(p<0.001)$ for participants with complete data and an aHR of $0.802(p<0.001)$ for all participants.

Conclusions: The treatment with fibrates in people with type 2 diabetes was independently associated with reduced progression to a first diagnosis of DR.

\section{INTRODUCTION}

Diabetic retinopathy (DR) remains a common diabetic complication globally, with an overall diabetes prevalence of approximately $35 \%$, varying according to the type of diabetes, ethnicity, diabetes duration, glycaemic control and blood pressure. ${ }^{1}$ In the UK, approximately $40 \%$ of people with type 2 diabetes are reported to have $\mathrm{DR}{ }^{2}$ In severe cases, DR can

\section{Strengths and limitations of this study}

- Routine data sources will also include missing data and measurements taken with varying periodicity.

- Owing to the nature of routine data, it was not possible to measure discrete stages of retinopathy.

- Confounding by indication may remain a source of bias.

result in blindness and represents the leading cause of blindness and visual disability in the non-elderly population. ${ }^{3}$ Furthermore, as the prevalence of diabetes has increased, rates of blindness attributed to DR have correspondingly increased. ${ }^{4}$

Early detection of DR in individuals with diabetes is critical in preventing visual loss and, consequently, DR screening programmes have been introduced throughout the UK. ${ }^{5-7}$ The control of diabetes-associated metabolic abnormalities such as dyslipidaemia is also considered important in preventing the progression of DR in people with type 2 diabetes. ${ }^{8}$ Recently, fenofibrate has shown promise in delaying DR progression in people with type 2 diabetes in the Fenofibrate Intervention and Event Lowering in Diabetes (FIELD) and The Action to Control Cardiovascular Risk in Diabetes (ACCORD) randomised controlled trials. ${ }^{9} 10$

In this study, we aimed to compare the rates of new onset of DR (first diagnosis of DR) in people with type 2 diabetes treated with fibrates and in matched non-exposed controls using UK population-based routine data.

\section{METHODS}

This retrospective, matched cohort study used data from the Clinical Practice Research Datalink (CPRD): a longitudinal, 
anonymised database derived from nearly 700 primary care practices throughout the UK that are broadly geographically and demographically representative of the country as a whole. ${ }^{11}$ At the time of the study, CPRD contained clinical records from over 11 million people. The data captured by CPRD include demographics, medical history, clinical investigations and drug prescriptions. The routine data are recorded electronically in general practice and monitored for quality by the UK Medicines and Healthcare products Regulatory Agency (MHRA). Diagnoses in CPRD are recorded using the Read code classification and have been validated in a number of studies, showing a high positive predictive value. $^{12}$

\section{Subject selection}

Study participants were classified as having type 2 diabetes if they had a diagnosis of diabetes and any of the following:

1. More than one diagnostic record exclusively for type 2 diabetes OR;

2. Prescription of two or more differing classes of oral antidiabetic drugs (OADs) OR;

3. A diagnostic code indicative of type 2 diabetes (regardless of conflicting diagnoses of type 1 or nonspecific diabetes) plus a prescription for an OAD.

Only those with a minimum of 180 days between practice registration and first diabetes presentation were included.

\section{Identification of individuals with fibrate therapy}

People initiating fibrate therapy following diabetes diagnosis were selected as the exposed group. Those participants with a DR event dated before the date of first fibrate or the date of diabetes presentation were excluded since this was a primary prevention study.

\section{Matching of controls}

Each member of the exposed cohort was matched at random with one person from the remaining diabetes population using the following criteria: gender, year of birth ( \pm 2 years), smoking status, duration of diabetes $( \pm 6$ months $)$, registration with a differing general practice (to minimise confounding by indication), prior statin use, systolic blood pressure $( \pm 5 \mathrm{~mm} \mathrm{Hg})$ and glycated haemoglobin (HbAlc; $\pm 0.5 \%$ ). Where either systolic blood pressure or HbAlc was missing, participants were matched on a control with the same missing value. The index date for the exposed patients was the date of first fibrate exposure.

\section{Study endpoints}

The primary endpoint for this study was first-recorded diagnosis of DR within the CPRD dataset. Relevant diagnoses and procedures as recorded by Read code in CPRD were assessed and identified by two clinicians experienced in diabetes-related eye disease. To investigate for competing risks, time to all-cause mortality and a combined endpoint of all-cause mortality or first DR were also characterised.

\section{Statistical analysis}

Descriptive analysis was performed, providing mean (SD) values for continuous variables and proportions for categorical variables. Time to clinical endpoints was compared using Cox proportional hazards models from index date to date of event or censorship. The selected potential covariates included age, gender, systolic blood pressure, baseline HbA1c, total cholesterol, body mass index and smoking status. Baseline morbidity was characterised in three ways: (1) history for each of cancer, large vessel disease, renal failure and visual deterioration; (2) the number of primary care contacts in the year prior to the index date; and (3) Charlson comorbidity index. ${ }^{13}$ Covariates were included in the final model where $p<0.20$. Participants with missing data were excluded automatically and the proportional hazards assumption was considered using evaluation of the Kaplan-Meier curves and analysis of the Schoenfeld residuals. Mean HbAlc per year with last observation carried forward was also modelled as a time-dependent variable.

\section{RESULTS}

\section{Subjects and baseline characteristics}

We identified 5038 eligible participants with a first exposure to fibrates. Three thousand one hundred and seventy-six (63\%) could be matched to one control, and, of these, $2599(81.8 \%)$ could be matched without any missing blood pressure or HbAlc values.

Baseline characteristics are detailed in table 1. There were significant differences between those exposed and not exposed to fibrates for total cholesterol (5.6 vs $4.7 \mathrm{mmol} / \mathrm{L})$, high-density lipoprotein cholesterol (1.1 vs $1.3 \mathrm{mmol} / \mathrm{L})$, low-density lipoprotein cholesterol (3.1 vs $2.6 \mathrm{mmol} / \mathrm{L}$ ), triglycerides (3.2 vs $1.9 \mathrm{mmol} / \mathrm{L}$ ) and primary care contacts in the preceding year (13.4 vs 12.6 consultations).

The most commonly used fibrate was bezafibrate, used by 1739 patients (54.8\%; table 2), followed by fenofibrate used by 1413 patients (44.5\%; table 2). The mean duration of fibrate therapy was 2.6 years (SD 2.8); median duration was 2.3 years (95\% CI 2.0 to 2.5$)$. Of note, fenofibrate was the fibrate with the longest use (mean duration 2.8 years; table 2 ). Time to discontinuation is shown in figure 1 .

\section{Crude event rates}

Four hundred and eighty-nine (15.4\%) patients exposed to fibrates developed a newly detected retinopathy compared with $569(17.9 \%)$ of those non-exposed. This represented a reduction in newly detected DR (33.4 events/1000 person-years vs 40.4 in non-exposed individuals; $\mathrm{p}=0.002)$ and in death or DR (50.6 vs 60.2, $\mathrm{p}<0.001$; table 3$)$. For participants matched with full 
Table 1 Baseline characteristics for all fibrate-exposed and matched fibrate and non-exposed controls

\begin{tabular}{|c|c|c|c|c|c|c|c|}
\hline \multirow{2}{*}{ Parameter } & \multicolumn{2}{|c|}{ All fibrate patients } & \multicolumn{2}{|c|}{ Fibrate matched } & \multicolumn{3}{|c|}{ Non-exposed controls } \\
\hline & 5038 & & 3176 & & 3176 & & \\
\hline Follow-up (years) (mean, SD) & 5.2 & (3.2) & 5.1 & $(3.2)$ & 5.0 & (3.2) & \\
\hline \multicolumn{8}{|l|}{ Matched variables } \\
\hline Age on index date (years) (mean, SD) & 60.9 & $(11.8)$ & 61.8 & $(10.7)$ & 61.8 & $(10.7)$ & \\
\hline $\begin{array}{l}\text { Age on diabetes presentation } \\
\text { (years) (mean, SD) }\end{array}$ & 57.1 & $(11.7)$ & 58.5 & $(10.4)$ & 58.6 & $(10.5)$ & \\
\hline Females $(n, \%)$ & 2203 & $(43.7 \%)$ & 1295 & $(40.8 \%)$ & 1295 & $(40.8 \%)$ & \\
\hline Duration of diabetes (years) (mean, SD) & 3.9 & (3.8) & 3.2 & (3.0) & 3.3 & (3.0) & \\
\hline Systolic BP (mm Hg) (mean, SD) & 137.8 & (16.5) & 136.5 & (13.4) & 136.5 & (13.4) & \\
\hline $\mathrm{HbA} 1 \mathrm{c}(\%, \mathrm{SD})$ & 7.4 & $(1.6)$ & 7.1 & $(1.2)$ & 7.1 & $(1.1)$ & \\
\hline Prior statin use $(n, \%)$ & 3781 & $(75.0 \%)$ & 2307 & $(72.6 \%)$ & 2307 & $(72.6 \%)$ & \\
\hline \multicolumn{7}{|l|}{ Unmatched variables } & $p$ Value \\
\hline BMI $\left(\mathrm{kg} / \mathrm{m}^{2}\right)$ (mean, SD) & 31.1 & $(5.4)$ & 30.7 & $(5.7)$ & 30.6 & (6.1) & 0.330 \\
\hline Diastolic BP (mm Hg) (mean, SD) & 79.4 & $(9.5)$ & 78.9 & $(9.1)$ & 78.7 & $(9.1)$ & 0.222 \\
\hline Total cholesterol (mmol/L) (mean, SD) & 5.6 & (1.3) & 5.6 & (1.4) & 4.7 & (1.1) & $<0.001$ \\
\hline $\mathrm{HDL}(\mathrm{mmol} / \mathrm{L})$ (mean, SD) & 1.2 & $(0.4)$ & 1.1 & $(0.4)$ & 1.3 & $(0.4)$ & $<0.001$ \\
\hline LDL (mmol/L) (mean, SD) & 3.1 & $(1.2)$ & 3.1 & (1.2) & 2.6 & (0.9) & $<0.001$ \\
\hline Triglycerides (mmol/L) (mean, SD) & 3.7 & $(2.4)$ & 3.2 & (1.6) & 1.9 & $(1.0)$ & $<0.001$ \\
\hline GP contacts in preceding year (mean, SD) & 13.9 & $(10.2)$ & 13.4 & (9.9) & 12.6 & (10.3) & 0.001 \\
\hline Charlson index (mean, SD) & 1.9 & $(1.3)$ & 2.0 & $(1.4)$ & 2.0 & $(1.4)$ & 0.404 \\
\hline
\end{tabular}

BMI, body mass index, BP, blood pressure; GP, general practitioner, HbA1c, glycated haemoglobin; HDL, high-density lipoprotein;

LDL, low-density lipoprotein.

systolic blood pressure and HbA1c data, the respective rate of DR was 34.3 events 1000 person-years for those treated with fibrates, versus 43.9 for controls $(p<0.001)$. For the combined endpoint of death or DR the respective rates were 51.2 and 63.4 events/1000 person-years $(\mathrm{p}<0.001)$. Figure 2 shows the Kaplan-Meier curves for each outcome. Of those coded with a retinopathy event, a majority in both groups had their first retinopathy event recorded as either background DR or retinopathy unspecified (95.1\% fibrate treated vs $96.1 \%$ non-fibrate treated).

\section{Adjusted survival}

After adjustment for baseline covariates and timedependent $\mathrm{HbA1c}$, time to DR was significantly greater for participants treated with fibrates, with an adjusted HR (aHR) of 0.785 (95\% CI 0.688 to $0.896 ; \mathrm{p}<0.001$ ) for those with a full dataset and $0.802(0.710$ to 0.905 ;

Table 2 Frequency and mean duration of specific fibrate use during follow-up period

\begin{tabular}{lcc}
\hline Fibrate & Number* & $\begin{array}{c}\text { Mean treatment } \\
\text { duration (years) }\end{array}$ \\
\hline Bezafibrate & 1739 & 2.1 \\
Fenofibrate & 1413 & 2.8 \\
Ciprofibrate & 187 & 2.3 \\
Gemfibrozil & 67 & 2.0 \\
Clofibrate & 1 & 0.1 \\
Any fibrate & 3176 & 2.6 \\
\hline *Patients may have received more than one type of fibrate during \\
follow-up.
\end{tabular}

$\mathrm{p}<0.001$ ) for all participants (table 4$)$. For the combined endpoint of death or DR the aHRs were 0.783 (0.689 to $0.886 ; \mathrm{p}<0.001)$ for those with a full dataset and 0.799 ( 0.724 to $0.883 ; \mathrm{p}<0.001)$ for all participants. There was no difference for death alone: $\mathrm{aHR}=0.910 \quad(0.760$ to $1.090 ; \mathrm{p}=0.305)$ and 0.889 (0.758 to $1.043 ; \mathrm{p}=0.149)$ for those with a full dataset and for all participants, respectively.

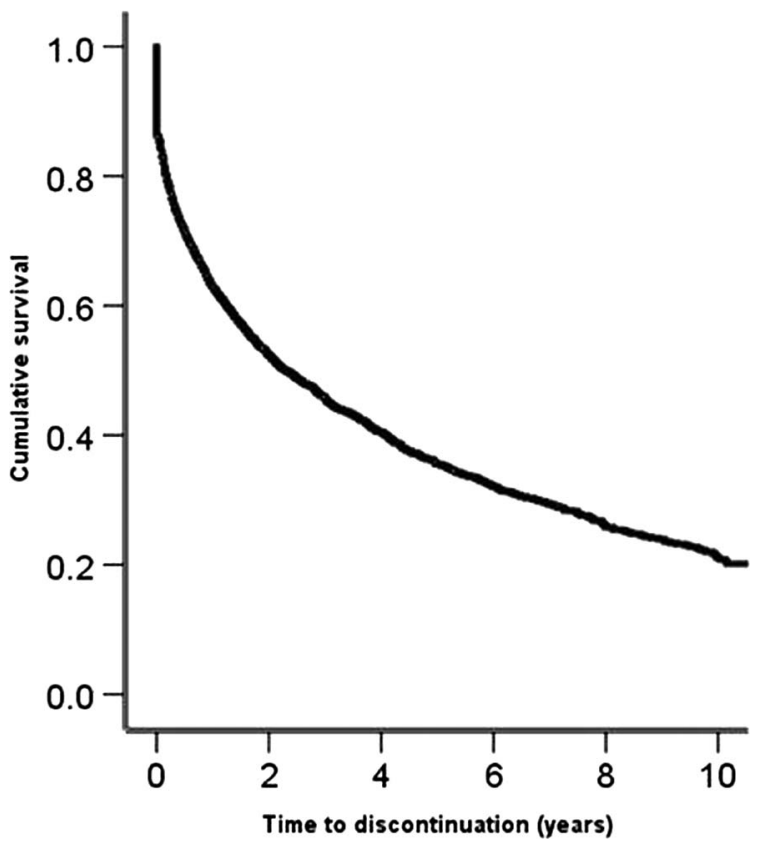

Figure 1 Time to discontinuation of fibrate. 
Table 3 Crude event frequency and event rates for fibrate-exposed and non-exposed subjects

\begin{tabular}{|c|c|c|c|c|c|}
\hline & \multicolumn{2}{|r|}{ Fibrate-exposed } & \multicolumn{2}{|r|}{ Non-exposed } & \multirow[b]{2}{*}{ p Value } \\
\hline & $\bar{n}$ & Rate/1000 person-year & $\bar{n}$ & Rate/1000 person-year & \\
\hline \multicolumn{6}{|l|}{ All patients } \\
\hline Diabetic retinopathy & 489 & 33.4 & 569 & 40.4 & 0.002 \\
\hline Death & 295 & 18.1 & 312 & 19.8 & 0.297 \\
\hline Death or diabetic retinopathy & 740 & 50.6 & 847 & 60.2 & $<0.001$ \\
\hline \multicolumn{6}{|l|}{ Patients with complete data } \\
\hline Diabetic retinopathy & 395 & 34.3 & 481 & 43.9 & $<0.001$ \\
\hline Death & 228 & 17.9 & 238 & 19.2 & 0.421 \\
\hline Death or diabetic retinopathy & 589 & 51.2 & 695 & 63.4 & $<0.001$ \\
\hline
\end{tabular}

\section{HbA1c and progression to DR}

HbA1c was modelled in three ways: as baseline values, time-dependent values and mean follow-up values. All three measures showed a general trend of increasing risk of DR with increasing HbAlc (figure 3).

\section{DISCUSSION}

Based on the current predictions, the diabetes pandemic will involve 552 million people, or $10 \%$ of the world's population by 2030, and in its wake the burden of complications is expected to increase. ${ }^{14}$ It is estimated that worldwide there are approximately 93 million people with DR, of whom 28 million have sightthreatening DR. ${ }^{1}$

This article reports an approximate $20 \%$ reduction in rates of first retinopathy for patients treated with fibrate therapy. Based on real-world observational data, this supports the findings from randomised controlled trials. The FIELD DR study, ${ }^{9}$ a randomised trial of monotherapy with $200 \mathrm{mg}$ micronised fenofibrate per day, showed a significant reduction in the need for laser therapy for sight-threatening lesions of macular oedema and proliferative DR in the fenofibrate group compared with the placebo group $(3.4 \%$ vs $4.9 \%$; $<<0.001)$. In a subpopulation with central grading of fundus photographs using adapted Early Treatment Diabetic Retinopathy Study (ETDRS) criteria, progression of DR was significantly reduced in participants with DR (ETDRS level 20 or more) at baseline.

The ACCORD Eye study provided confirmatory evidence: fenofibrate (160 mg tablet per day, bioequivalent to the FIELD study's $200 \mathrm{mg}$ micronized capsule) when added to a statin (simvastatin) slowed the progression of DR compared with simvastatin plus placebo, with an OR of 0.60 in the primary endpoint of three or more steps of progression on the ETDRS scale or a need for laser therapy or vitrectomy to treat proliferative DR. ${ }^{10}$

In neither the FIELD nor the ACCORD studies did the mechanism of the effect appear attributable to the observed changes in the circulating lipid profiles. Other clinical evidence linking dyslipidaemia and DR has been variable. ${ }^{15-19}$ The impact of lipid-lowering therapy with statins has been shown to retard the progression of retinopathy in those with hypercholesterolaemia, ${ }^{20}$ although a positive outcome has not always been evident. ${ }^{21}$
Figure 2 Kaplan-Meier (unadjusted) curves for time to clinical endpoints.
Diabetic retinopathy

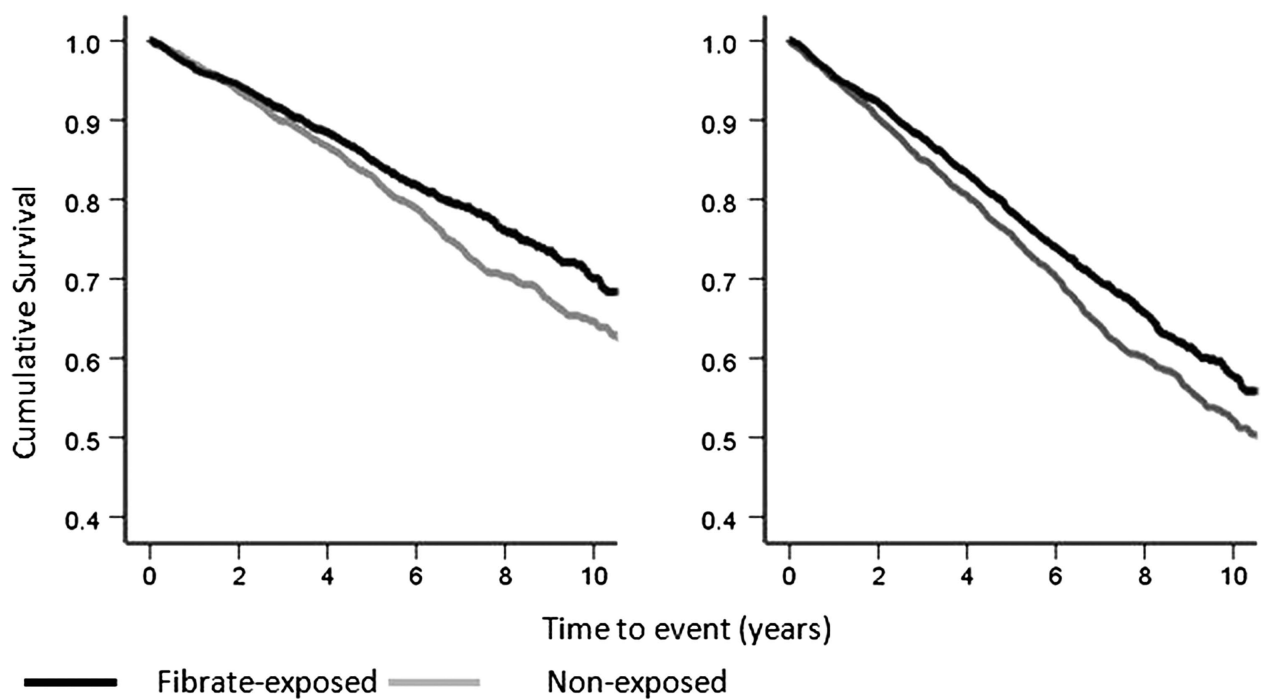


Table 4 Adjusted Cox models characterising progression to the respective clinical endpoints of newly diagnosed diabetic retinopathy and all-cause mortality

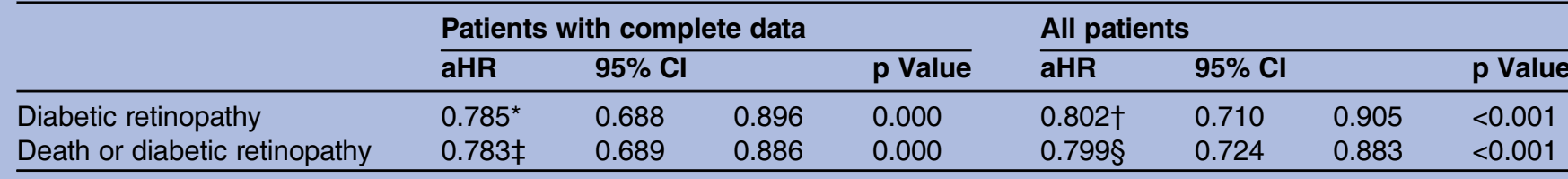

${ }^{*}$ Baseline $\mathrm{HbA1c}$, diabetes duration.

$\ddagger A g e$, gender, smoking status, baseline $\mathrm{HbA1c}$, prior primary care contacts, diabetes duration and Charlson index.

†Time-dependent $\mathrm{HbA1c}$, diabetes duration.

$\S A g e$, smoking status, prior primary care contacts, Charlson index, time-dependent $\mathrm{HbA} 1 \mathrm{c}$ and diabetes duration.

aHR, adjusted HR; HbA1c, glycated haemoglobin.

The mechanism(s) for the changes observed in this study may or may not be related to the lipid-lowering effects of fibrates. ${ }^{22}$ While there is a little evidence to link the quantitative lowering of the major commonly measured circulating lipid fractions, ${ }^{9} 10$ qualitative changes may occur. Fibrates also cause an increase in apolipoprotein A1 (apoA1) expression, which could limit the lipotoxicity at the level of the retina, in addition to its inherent potent antioxidant and antiinflammatory properties. ${ }^{23-25}$

Non-lipid-related mechanisms are currently regarded as the most plausible explanation for the beneficial effect of fibrate on DR. Fibric acid derivatives possess independent anti-inflammatory and antioxidant properties mediated by their proliferator-activated receptor- $\alpha$ (PPAR- $\alpha$ ) agonist activity. Inflammation is decreased via inhibition of enhanced nuclear transcription factor-kappaB (NF-Kb) activity, thus preventing interleukin (IL)-1-induced expression of IL-6 and cyclo-oxygenase- 2 and in turn preventing any increase in retinal capillary permeability at an early stage in the evolution of DR. ${ }^{26} 27$ Glucolipotoxicity-induced NF-kB activation can also increase the expression of the

HbA1c and progression to diabetic retinopathy*

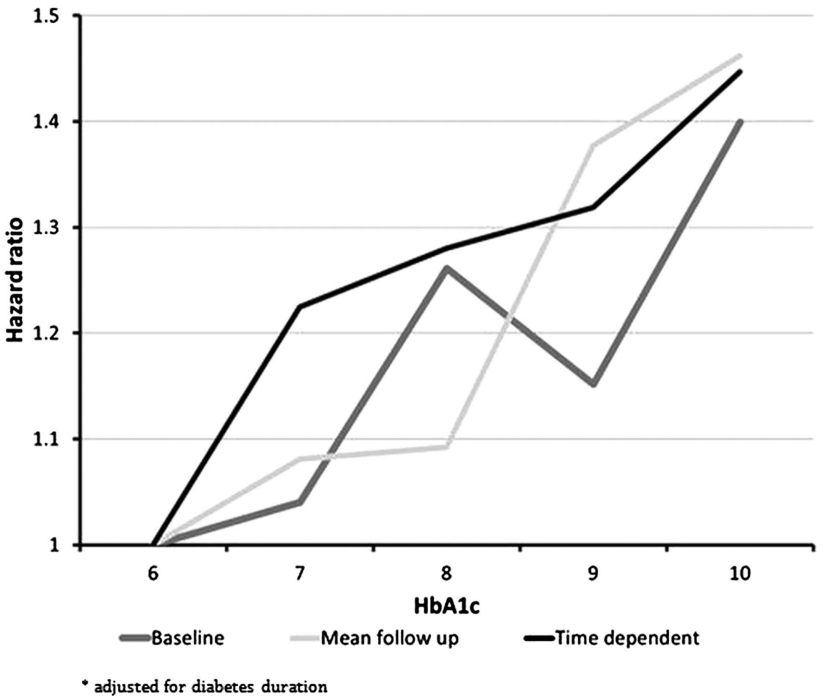

Figure 3 Glycated haemoglobin $(\mathrm{HbA} 1 \mathrm{c})$ and progression to diabetic retinopathy*. proinflammatory cytokine $\mathrm{TNF}-\alpha$, resulting in the upregulation of adhesion molecules, leucocyte and monocyte activation and vessel loss. ${ }^{28} 29$ Fenofibric acid is also known to prevent the disruption of retinal pigment epithelium (RPE) cells by the stress-induced cytokine IL-1 $\beta$, achieved through the suppression of AMP-activated protein kinase activation. $^{30}$ In addition, further protection by fenofibric acid of the RPE may occur through the induction of autophagy and insulin-like growth factor-1 receptor mediated survival (antiapoptotic) pathways. $^{31}$ Fenofibric acid has also been demonstrated to prevent the increased breakdown of the blood-brain capillary barrier by lowering the overexpression of fibronectin and collagen IV basement membrane components of the RPE cells. ${ }^{32}$ Fibric acid can also downregulate the expression of vascular endothelial growth factor receptor $2,,^{33}$ a key regulator of vascular regrowth in response to retinal hypoxia, ${ }^{34}$ thus avoiding an excess neovascularisation resulting in proliferative DR. ${ }^{35} 36$ Fibrates, by virtue of their PPAR- $\alpha$ agonist properties, can induce the expression of nitric oxide synthase and decrease cellular adhesion molecules, thereby inhibiting NF-Kb and suppressing the genes that encode adhesion molecules. ${ }^{37}$ They also possess neuroprotective properties. ${ }^{38}$

These cumulative observations could well explain the retardation in DR progression found in previous studies $^{9} 10$ and the prevention of DR onset found in this study in participants with type 2 diabetes. Despite the initial and subsequent reversible elevation of creatinine during treatment with fenofibrate, a reduction in albuminuria and a slower decline in the estimated glomerular filtration rate (GFR) over 5 years are reassuring. ${ }^{39}$ A greater estimated GFR preservation was seen in those with baseline hypertriglyceridaemia or dyslipidaemia compared with those without. In those on fenofibrate, benefit was also related to the lipid-lowering response.

This study has a number of limitations. Routine data such as CPRD is able to complement randomised controlled trials evidence as it is based on real-world experience in the populations who are receiving the treatment rather than highly selected patients who may be less morbid and more compliant with the treatment regimens under trial conditions. However, there may be issues concerning the data quality. In this particular study, the data lacked a sufficient granularity to allow progression to discrete stages of retinopathy to be adequately captured, and 
thus we used the criterion of progression from no recorded retinopathy to first event as our primary outcome.

Routine data sources will also include missing data and measurements taken with varying periodicity, sometimes involving key variables that may be important as covariates. We therefore compared the results from sets of exposed and non-exposed patients matched using two different techniques: the first requiring full data values for all the match criteria and the second allowing missing data values for systolic blood pressure and HbA1c, where participants were then matched with a control with the same missing data value. There was no substantial difference in the HRs observed in either set.

It is also accepted that there will be additional coding imperfections, lack of standardisation of biochemical measures (such as HbAlc) and variations between biochemical test centres. There is no reason to suppose that there was a coding bias between the exposed and non-exposed groups, but this should be considered as a potential bias in the interpretation of the results. Equally, confounding by indication will also be considered as a source of bias.

The patient ethnicity, known to impact on diabetes progression and outcome, was not systematically recorded within CPRD. Exposure to fibrates and to other lipidlowering pharmacotherapies of interest can only be taken as an intention to treat on the part of the prescriber. Fenofibrate was the second most common prescribed fibrate in our study but with the longest duration of exposure. However, we have no data as to whether the patient actually filled the prescription at the pharmacy or, further, as to whether they took the medicine at the recorded dosage.

In conclusion, the evidence from most common-scale randomised studies (the FIELD and ACCORD Eye studies) supports the use of the fibric acid derivative fenofibrate as an adjunctive therapy to maintaining good glycaemic and blood pressure control, in an effort to prevent the progression and enhance the regression of DR in people with type 2 diabetes. These new data suggest that the introduction of fenofibrate may be valuable in preventing the expression of DR. Randomised studies are necessary to consolidate or refute these findings.

\author{
Author affiliations \\ ${ }^{1}$ Department of Primary Care and Public Health, School of Medicine, Cardiff \\ University, The Pharma Research Centre, Cardiff Medicentre, Cardiff, UK \\ ${ }^{2}$ Centre for Endocrine and Diabetes Sciences, University Hospital of Wales, \\ Cardiff, UK \\ ${ }^{3}$ Abbott Products Operations AG, Allschwil, Switzerland \\ ${ }^{4}$ Department of Global Epidemiology, Pharmatelligence, Cardiff, UK
}

Contributors All the coauthors were involved with the study design. SJ-J carried out the data extraction and matching. CLM carried out the majority of the data analysis. CDP contributed to the data analysis and the interpretation of the study. DRO contributed to the interpretation of the study and the writing of the article. CJC wrote the first draft of the article and all coauthors were then involved editorially. CJC is the guarantor.

Funding This work was supported by Abbott Products Operations AG. Coauthors from the funding body helped design the study and suggested editorial changes.
Competing interests ESMC and PA are employees of Abbott Products Operations AG; CLM and SJ-J are employees of a research consultancy receiving funding from pharmaceutical companies; CJC has received research grants from various health-related organisations, including Abbott, Astellas Pharma Inc, Diabetes UK, Eli Lilly, the Engineering and Physical Sciences Research Council, the European Association for the Study of Diabetes, Ferring Pharmaceuticals, GlaxoSmithKline plc, the Medical Research Council, Medtronic, Merck KGaA, the National Health Service, Pfizer Ltd, Sanofi UK, Shire Pharmaceuticals Ltd and Wyeth Pharmaceuticals; he also consults for Amylin Pharmaceuticals LLC, Aryx Therapeutics Inc, Astellas, Boehringer Ingelheim GmbH, Bristol-Myers Squibb, Diabetes UK, Eisai Co Ltd, Eli Lilly, Ferring, GlaxoSmithKline, Ipsen Ltd, Medtronic Inc, Merck, Pfizer, Sanofi, Takeda Pharmaceutical Co and Wyeth; CDP consults for Astellas, Eli Lilly, Ferring, Medtronic, Novo Nordisk A/S, Sanofi and Wyeth.

Ethics approval Studies using the CPRD are covered by ethics approval granted by Trent Multicentre Research Ethics Committee (reference 05/ MRE04/87). This study was granted CPRD Independent Scientific Advisory Committee approval (ISAC 12-130).

Provenance and peer review Not commissioned; externally peer reviewed.

Data sharing statement Clinical Practice Research Datalink (CPRD) is an open data source and all authors had access to all of the data and can take responsibility for the integrity of the data and the accuracy of the data analysis.

Open Access This is an Open Access article distributed in accordance with the Creative Commons Attribution Non Commercial (CC BY-NC 3.0) license, which permits others to distribute, remix, adapt, build upon this work noncommercially, and license their derivative works on different terms, provided the original work is properly cited and the use is non-commercial. See: http:// creativecommons.org/licenses/by-nc/3.0/

\section{REFERENCES}

1. Yau JW, Rogers SL, Kawasaki R, et al. Global prevalence and major risk factors of diabetic retinopathy. Diabetes Care 2012;35:556-64.

2. Sivaprasad S, Gupta B, Gulliford MC, et al. Ethnic variation in the prevalence of visual impairment in people attending diabetic retinopathy screening in the United Kingdom (DRIVE UK). PLoS ONE 2012;7:e39608.

3. Kohner E, Allwinkle J, Andrews J, et al. Saint Vincent and improving diabetes care: report of the Visual Handicap Group. Diabet Med 1996;13(Suppl 4):S13-26.

4. Bunce $C$, Wormald R. Leading causes of certification for blindness and partial sight in England \& Wales. BMC Public Health 2006;6:58.

5. Scanlon $\mathrm{PH}$. The English national screening programme for sight-threatening diabetic retinopathy. J Med Screen 2008;15:1-4.

6. Leese GP, Morris AD, Olson J. A national retinal screening programme for diabetes in Scotland. Diabet Med 2003;20:962-4.

7. Thomas RL, Dunstan F, Luzio SD, et al. Incidence of diabetic retinopathy in people with type 2 diabetes mellitus attending the Diabetic Retinopathy Screening Service for Wales: retrospective analysis. BMJ 2012;344:e874.

8. Lim LS, Wong TY. Lipids and diabetic retinopathy. Expert Opin Biol Ther 2011;12:93-105.

9. Keech AC, Mitchell P, Summanen PA, et al. Effect of fenofibrate on the need for laser treatment for diabetic retinopathy (FIELD study): a randomised controlled trial. Lancet 2007;370:1687-97.

10. ACCORD Study Group and ACCORD Eye Study Group. Effects of medical therapies on retinopathy progression in type 2 diabetes. N Engl J Med 2010;363:233-44.

11. Medicines and Healthcare products Regulatory Agency. The Clinical Practice Research Datalink. 2012. http://www.cprd.com

12. Herrett E, Thomas SL, Schoonen WM, et al. Validation and validity of diagnoses in the General Practice Research Database: a systematic review. Br J Clin Pharmacol 2010;69:4-14.

13. Charlson ME, Pompei P, Ales KL, et al. A new method of classifying prognostic comorbidity in longitudinal studies: development and validation. J Chronic Dis 1987;40:373-83.

14. International Diabetes Federation. IDF Diabetes Atlas. 5th edn. Brussels, Belgium: International Diabetes Federation, 2011. http:// www.idf.org/diabetesatlas 
15. Klein BE, Moss SE, Klein R, et al. The Wisconsin epidemiologic study of diabetic retinopathy. XIII. Relationship of serum cholesterol to retinopathy and hard exudate. Ophthalmology 1991;98:1261-5.

16. Chew EY, Klein ML, Ferris FL III, et al. Association of elevated serum lipid levels with retinal hard exudate in diabetic retinopathy. Early Treatment Diabetic Retinopathy Study (ETDRS) Report 22. Arch Ophthalmol 1996;114:1079-84.

17. Wong TY, Cheung N, Tay WT, et al. Prevalence and risk factors for diabetic retinopathy: the Singapore Malay Eye Study. Ophthalmology 2008;115:1869-75.

18. Lyons TJ, Jenkins AJ, Zheng D, et al. Diabetic retinopathy and serum lipoprotein subclasses in the DCCT/EDIC cohort. Invest Ophthalmol Vis Sci 2004;45:910-18.

19. Rema M, Srivastava BK, Anitha B, et al. Association of serum lipids with diabetic retinopathy in urban south Indians-the Chennai Urban Rural Epidemiology Study (CURES) Eye Study-2. Diabet Med 2006;23:1029-36.

20. Gupta A, Gupta V, Thapar S, et al. Lipid-lowering drug atorvastatin as an adjunct in the management of diabetic macular edema. $A m \mathrm{~J}$ Ophthalmol 2004;137:675-82.

21. Colhoun HM, Betteridge DJ, Durrington PN, et al. Primary prevention of cardiovascular disease with atorvastatin in type 2 diabetes in the Collaborative Atorvastatin Diabetes Study (CARDS): multicentre randomised placebo-controlled trial. Lancet 2004;364:685-96.

22. Wong TY, Simó R, Mitchell P. Fenofibrate-a potential systemic treatment for diabetic retinopathy? Am J Ophthalmol 2012;154:6-12.

23. Barter PJ, Nicholls S, Rye KA, et al. Antiinflammatory properties of HDL. Circ Res 2004;95:764-72.

24. Tserentsoodol N, Gordiyenko NV, Pascual I, et al. Intraretinal lipid transport is dependent on high density lipoprotein-like particles and class B scavenger receptors. Mol Vis 2006;12:1319-33.

25. Simó R, García-Ramírez M, Higuera M, et al. Apolipoprotein A1 is overexpressed in the retina of diabetic patients. Am J Ophthalmol 2009;147:319-25.

26. Poynter ME, Daynes RA. Peroxisome proliferator-activated receptor alpha activation modulates cellular redox status, represses nuclear factor-kappaB signaling, and reduces inflammatory cytokine production in aging. J Biol Chem 1998;273:32833-41.

27. Delerive P, De Bosscher K, Besnard S, et al. Peroxisome proliferator-activated receptor alpha negatively regulates the vascular inflammatory gene response by negative cross-talk with transcription factors NF-kappaB and AP-1. J Biol Chem 1999;274:32048-54.
28. Marx N, Sukhova GK, Collins T, et al. PPARalpha activators inhibit cytokine-induced vascular cell adhesion molecule-1 expression in human endothelial cells. Circulation 1999;

99:3125-31.

29. Hiukka A, Maranghi M, Matikainen N, et al. PPARalpha: an emerging therapeutic target in diabetic microvascular damage. Nat Rev Endocrinol 2010;6:454-63.

30. Villarroel M, Garcia-Ramírez M, Corraliza L, et al. Fenofibric acid prevents retinal pigment epithelium disruption induced by interleukin-1 13 by suppressing AMP-activated protein kinase (AMPK) activation. Diabetologia 2011;54:1543-53.

31. Miranda S, González-Rodríguez Á, García-Ramírez M, et al. Beneficial effects of fenofibrate in retinal pigment epithelium by the modulation of stress and survival signaling under diabetic conditions. J Cell Physiol 2012;227:2352-62.

32. Trudeau K, Roy S, Guo W, et al. Fenofibric acid reduces fibronectin and collagen type IV overexpression in human retinal pigment epithelial cells grown in conditions mimicking the diabetic milieu: functional implications in retinal permeability. Invest Ophthalmol Vis Sci 2011;52:6348-54.

33. Meissner M, Stein M, Urbich $\mathrm{C}$, et al. PPARalpha activators inhibit vascular endothelial growth factor receptor-2 expression by repressing Sp1-dependent DNA binding and transactivation. Circ Res 2004;94:324-32.

34. Sapieha P, Hamel D, Shao Z, et al. Proliferative retinopathies: angiogenesis that blinds. Int J Biochem Cell Biol 2010;42:5-12.

35. Fisslthaler $B$, Fleming I. Activation and signaling by the AMP-activated protein kinase in endothelial cells. Circ Res 2009;105:114-27

36. Kim J, Ahn JH, Kim JH, et al. Fenofibrate regulates retinal endothelial cell survival through the AMPK signal transduction pathway. Exp Eye Res 2007;84:886-93.

37. Okayasu T, Tomizawa A, Suzuki K, et al. PPARalpha activators upregulate eNOS activity and inhibit cytokine-induced NF-kappaB activation through AMP-activated protein kinase activation. Life $\mathrm{Scl}$ 2008;82:884-91.

38. Bordet R, Ouk T, Petrault $\mathrm{O}$, et al. PPAR: a new pharmacological target for neuroprotection in stroke and neurodegenerative diseases. Biochem Soc Trans 2006;34:1341-6.

39. Davis TM, Ting R, Best JD, et al. Effects of fenofibrate on renal function in patients with type 2 diabetes mellitus: the Fenofibrate Intervention and Event Lowering in Diabetes (FIELD) Study. Diabetologia 2011;54:280-90. 